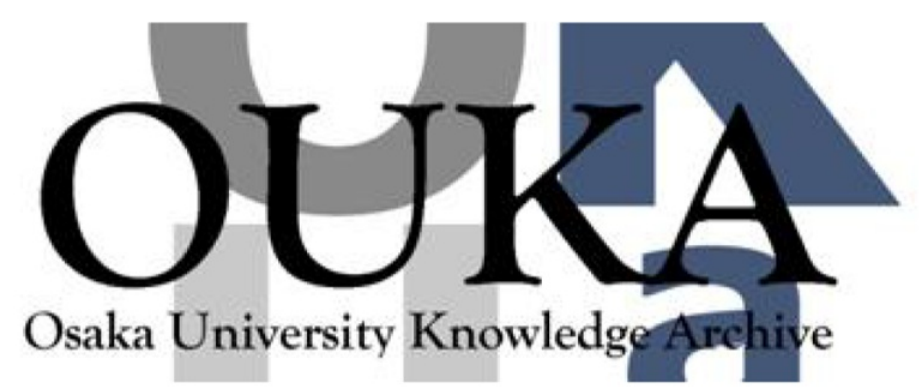

\begin{tabular}{|c|l|}
\hline Title & $\begin{array}{l}\text { Laser generated proton beam focusing and high } \\
\text { temperature isochoric heating of solid matter }\end{array}$ \\
\hline Author(s) & Snavely, R. A.; Zhang, B.; Akli, K. et al. \\
\hline Citation & Physics of Plasmas. 14(9) p. 092703 \\
\hline Issue Date & $2007-09$ \\
\hline oaire:version & VoR \\
\hline URL & https://hdl. handle. net/11094/3087 \\
\hline rights & \\
\hline Note & \\
\hline
\end{tabular}

Osaka University Knowledge Archive : OUKA

https://ir. Library. osaka-u. ac. jp/

Osaka University 


\section{AIP Plasmas}

\section{Laser generated proton beam focusing and high temperature isochoric heating of solid matter}

R. A. Snavely, B. Zhang, K. Akli, Z. Chen, R. R. Freeman et al.

Citation: Phys. Plasmas 14, 092703 (2007); doi: 10.1063/1.2774001

View online: http://dx.doi.org/10.1063/1.2774001

View Table of Contents: http://pop.aip.org/resource/1/PHPAEN/v14/i9

Published by the American Institute of Physics.

\section{Related Articles}

Simultaneous generation of ions and high-order harmonics from thin conjugated polymer foil irradiated with ultrahigh contrast laser

Appl. Phys. Lett. 99, 181501 (2011)

Production of neutrons up to $18 \mathrm{MeV}$ in high-intensity, short-pulse laser matter interactions

Phys. Plasmas 18, 100703 (2011)

Directional elliptically polarized terahertz emission from air plasma produced by circularly polarized intense femtosecond laser pulses

Appl. Phys. Lett. 99, 161505 (2011)

Enhancements of extreme ultraviolet emission using prepulsed $\mathrm{Sn}$ laser-produced plasmas for advanced lithography applications

J. Appl. Phys. 110, 083303 (2011)

Isothermal, mass-limited rarefactions in planar and spherical geometry

Phys. Plasmas 18, 104506 (2011)

\section{Additional information on Phys. Plasmas}

Journal Homepage: http://pop.aip.org/

Journal Information: http://pop.aip.org/about/about_the_journal

Top downloads: http://pop.aip.org/features/most_downloaded

Information for Authors: http://pop.aip.org/authors

\section{ADVERTISEMENT}

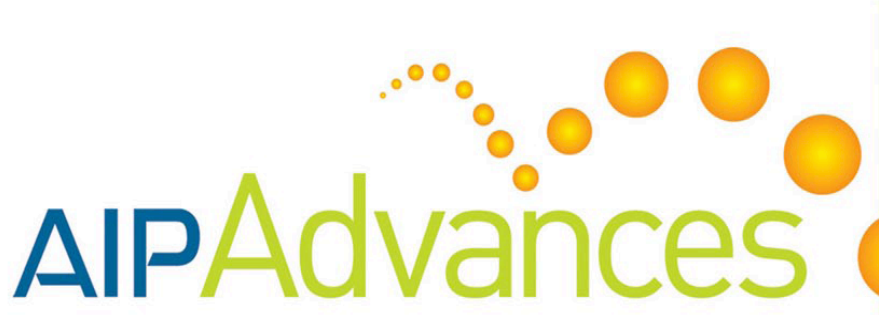

Submit Now

Explore AIP's new

open-access journal

Article-level metrics now available

Join the conversation!

Rate \& comment on articles 


\title{
Laser generated proton beam focusing and high temperature isochoric heating of solid matter
}

\author{
R. A. Snavely, ${ }^{1}$ B. Zhang, ${ }^{2, a)}$ K. Aklii, ${ }^{2}$ Z. Chen, ${ }^{3}$ R. R. Freeman, ${ }^{4}$ P. Gu, ${ }^{4}$ S. P. Hatchett,${ }^{1}$ \\ D. Hey, ${ }^{2}$ J. Hill, ${ }^{4}$ M. H. Key, ${ }^{1}$ Y. Izawa, ${ }^{3}$ J. King, ${ }^{2}$ Y. Kitagawa, ${ }^{3}$ R. Kodama, ${ }^{3}$ \\ A. B. Langdon, ${ }^{1}$ B. F. Lasinski, ${ }^{1}$ A. Lei, ${ }^{3}$ A. J. MacKinnon, ${ }^{1}$ P. Patel, ${ }^{1}$ R. Stephens, ${ }^{5}$ \\ M. Tampo, ${ }^{3}$ K. A. Tanaka, ${ }^{3}$ R. Town, ${ }^{1}$ Y. Toyama, ${ }^{3}$ T. Tsutsumi, ${ }^{3}$ S. C. Wilks, ${ }^{1}$ T. Yabuuchi, ${ }^{3}$ \\ and J. Zheng ${ }^{3}$ \\ ${ }^{1}$ University of California, Lawrence Livermore National Laboratory, P.O. Box 808, Livermore, \\ California 14550, USA \\ ${ }^{2}$ University of California, Davis, Department of Applied Science, P.O. Box 808, L714, Livermore, \\ California 94552 USA \\ ${ }^{3}$ Institute of Laser Energetics, Osaka University, Suita, Osaka 565, Japan \\ ${ }^{4}$ The Ohio State University, Columbus, Ohio 14320 USA \\ ${ }^{5}$ General Atomics, San Diego, California 13286 USA
}

(Received 2 January 2007; accepted 18 July 2007; published online 21 September 2007)

\begin{abstract}
The results of laser-driven proton beam focusing and heating with a high energy (170 J) short pulse are reported. Thin hemispherical aluminum shells are illuminated with the Gekko petawatt laser using $1 \mu \mathrm{m}$ light at intensities of $\sim 3 \times 10^{18} \mathrm{~W} / \mathrm{cm}^{2}$ and measured heating of thin $\mathrm{Al}$ slabs. The heating pattern is inferred by imaging visible and extreme-ultraviolet light Planckian emission from the rear surface. When Al slabs $100 \mu \mathrm{m}$ thick were placed at distances spanning the proton focus beam waist, the highest temperatures were produced at $0.94 \times$ the hemisphere radius beyond the equatorial plane. Isochoric heating temperatures reached $81 \mathrm{eV}$ in $15 \mu \mathrm{m}$ thick foils. The heating with a three-dimensional Monte Carlo model of proton transport with self-consistent heating and proton stopping in hot plasma was modeled. (C) 2007 American Institute of Physics.
\end{abstract}

[DOI: $10.1063 / 1.2774001]$

\section{INTRODUCTION}

Fast ignition (FI) of thermonuclear fusion is an attractive scheme to increase the energy gain, reduce the laser driver energy, and relax fuel compression uniformity in inertial confinement fusion. ${ }^{1}$ Recent work has demonstrated $>20 \%$ efficient coupling of picosecond pulse laser energy to a compressed core through fast electrons. ${ }^{2}$ The transport of energy by the electrons is subject to complex effects from selfconsistent $E$ and $B$ fields, which could reduce the efficiency in full-scale fast ignition.

Shortly after the first observations of collimated lasergenerated proton plasma jets ${ }^{3}$ it was suggested that protons could offer an alternative path to FI with simpler energy transport physics. ${ }^{4,5}$ While there are several mechanisms of laser acceleration of protons, ${ }^{6-10}$ that leading to focusing is proton plasma expansion driven by hot electron pressure ${ }^{11}$ perpendicular to a concave surface. The first demonstration of generation of a focused proton plasma jet from the inner surface of a thin tightly curved hemi-shell and of isochoric heating (in time short relative to hydrodynamic expansion time) ${ }^{12}$ was made using a $10 \mathrm{~J}, 0.1 \mathrm{ps}$ laser system. ${ }^{13}$ The results suggest both that proton isochoric heating offers new possibilities in high energy density physics through creating unique states of matter and that fast ignition with protons merits further study.

Modeling predicts that the FI requirement at a typical FI deuterium tritium fuel density of $300 \mathrm{~g} / \mathrm{cm}^{3}$ is to deliver

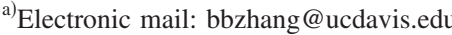

$20 \mathrm{~kJ}$ of protons into $40 \mu \mathrm{m}$ diameter spot with a suitable Boltzmann-like energy spectrum $(k T=3$ to $5 \mathrm{MeV}) \cdot{ }^{14-16}$ For a practical feasibility the laser to hot spot energy coupling efficiency should be $>10 \%$. The fact that conversion of short pulse laser energy to proton beam energy has reached efficiencies $>10 \%^{3,11}$ is encouraging.

\section{EXPERIMENTAL CONFIGURATION}

Here we describe proton focusing experiments with $20 \times$ higher energy than was used in the initial experiments. We used the Gekko petawatt (PW) Laser System at ILEOsaka University. ${ }^{17}$ It delivered pulses of 700 fs duration with an average of $170 \mathrm{~J}$ on target. The targets had two parts illustrated in Fig. 1: a hemispherical Al shell $360 \mu \mathrm{m}$ diameter with 13-16 $\mu \mathrm{m}$ thickness, which was illuminated by the laser pulse on its exterior pole and generated the proton beam from its inner shell surface, and an Al slab of thickness 15 to $100 \mu \mathrm{m}$ for proton beam stopping and heating mounted close to the hemisphere on a stalk. The laser was focused with an $f / 10$ mirror with a spot size of $40 \times 60 \mu \mathrm{m}$ estimated from x-ray pinhole camera images, giving an intensity of $\sim 3 \times 10^{18} \mathrm{~W} / \mathrm{cm}^{2}$.

Three main diagnostics were used, as shown in Fig. 1: two imaged the Planckian emission from the rear surface of the heated foil in the visible and extreme-ultraviolet light (XUV). A time-integrated XUV imaging microscope ${ }^{18}$ had a band pass of $8 \mathrm{eV}$ centered at $68 \mathrm{eV}$. It used $\mathrm{Mo}_{2} \mathrm{C}: \mathrm{Si}$ multilayer mirrors. The focal length of the spherical primary 


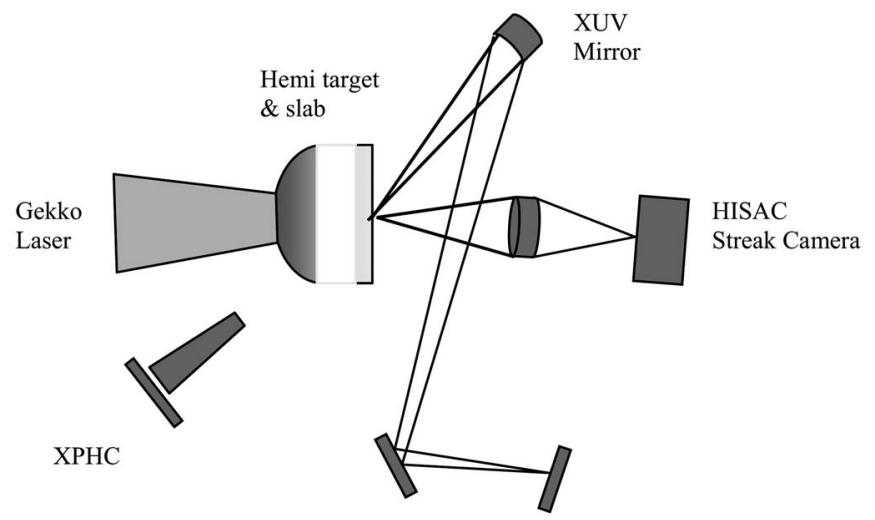

FIG. 1. Schematic of experiment. Positions of XUV, HISAC, and x-ray pinhole cameras.

mirror was $308 \mathrm{~mm}$ and a $45^{\circ}$ flat turning mirror was included for stray light suppression.

The XUV magnification was 10.2 or 13.1 and the measured resolution was $<10 \mu \mathrm{m}$. The viewing angle was $41^{\circ}$ from the normal to the rear target surface. A Princeton Instruments cooled CCD camera (SITE CCD back-thinned and back-illuminated $1 \mathrm{k} \times 1 \mathrm{k}$ ) recorded the XUV image. The XUV imaging channel was absolutely calibrated. ${ }^{18}$ The $68 \mathrm{eV}$ mirrors were calibrated with synchrotron radiation source at Brookhaven National Laboratory. Mirror reflectivity was obtained with a pair of diodes measuring the incoming XUV and the reflection off the mirrors. An Fe55 radioactive source was used to calibrate CCD cameras. The number of counts on a pixel produced by absorption of a photon at the CCD camera was carefully measured.

A high-speed optical imaging streak camera (HISAC) imaged the rear surface at $15^{\circ}$ from the normal in the spectral range $400-700 \mathrm{~nm}$. A notch filter between 500 and $550 \mathrm{~nm}$ was used to block imaging of $2 \omega$ transition radiation. It produces gated images in a $10 \times 10$ pixel array with $45 \mu \mathrm{m}$ spatial resolution and 50 ps temporal resolution using a multichannel fiber optic technique. ${ }^{19}$ The images are constructed by two-dimensional (2D) interpolation between pixels from the optical streak signal. Thus, the HISAC images were obtained from the first $50 \mathrm{ps}$ of Planckian emission.

A time-integrated x-ray pinhole camera with a $20 \mu \mathrm{m}$ diameter aperture and a $20 \mu \mathrm{m}$ thick Be filter imaged the $\mathrm{x}$ rays from the front of the laser target interaction region at an angle of $35^{\circ}$ to the laser.

\section{EXPERIMENTAL RESULTS}

To study the focusing, we varied two parameters for this experiment. Either we moved a $100 \mu \mathrm{m}$ thick Al slab along the axis within the focal region of the proton plasma jet or we fixed the distance and varied the Al thickness.

We used a simple model based on measured proton source diameters and proton plasma jet divergence measurements from planar foil targets to infer the location of the best focus from a spherically bent foil. The model assumed the angle of the flow relative to the normal to the surface as a function of the radius is the same in both cases. ${ }^{13}$ For the focusing study, the placement of the $\mathrm{Al}$ slab [the side toward
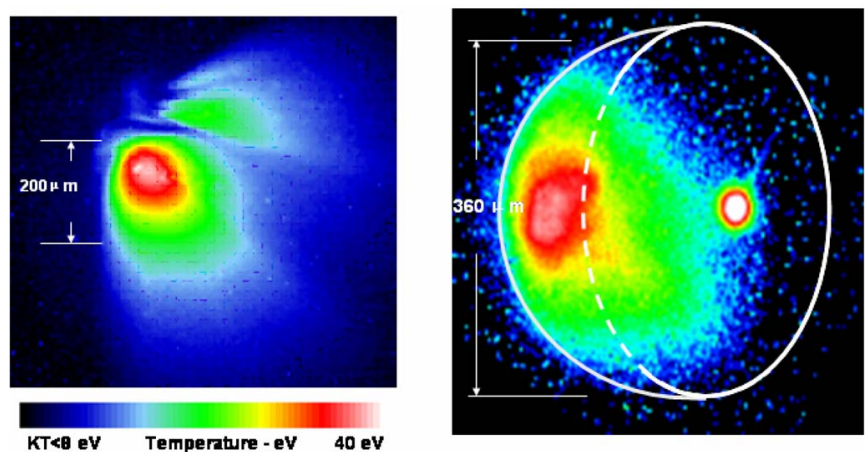

FIG. 2. (Color online) (Left) XUV image of proton heating of a $60 \mu \mathrm{m} \mathrm{Al}$ foil at $D / r=1.5$, converted to temperature. (Right) X-ray pinhole camera image of a laser irradiated $\mathrm{Al}$ hemisphere with a $100 \mu \mathrm{m}$ thick $\mathrm{Al}$ foil at $D / r=1.8$.

the hemi-shell] relative to the pole of the hemisphere was in the range 1.25 to 2.5 times the hemisphere radius. We characterize the targets with this ratio $D / r$, where $D$ is the distance from the apex of the hemi-shell to the rear surface of the secondary $\mathrm{Al}$ foil and $r$ is the radius of the hemisphere.

Typical analyzed data from the XUV microscope are shown in Fig. 2 (left). The $41^{\circ}$ oblique view of the heated region shows a bright XUV emission spot and a weaker secondary spot, converted to a map of temperature with a peak of $42 \mathrm{eV}$ and a sensitivity floor of $8 \mathrm{eV}$. There is also an expansion plume to the right along the target normal direction due to the oblique view and time-integrated XUV image acquisition. The image is transformed into temperature via a numerical model and absolute calibration discussed later. Figure 2 (right) shows an x-ray pinhole camera image. The $40 \times 60 \mu \mathrm{m}$ laser focal spot is seen in the upper left. The size is consistent with previous spot measurements. Electron refluxing in the hemisphere produces thermal x-rays over a broader region than the laser focal spot outlining the hemisphere. An intense saturated spot (radius $<20 \mu \mathrm{m}$ ) is seen in the proton focal region on the front surface of the $100 \mu \mathrm{m}$ thick Al slab. This image is recorded in transmission of $\mathrm{x}$ rays through the $\mathrm{Al}$ hemispherical shell laser target and implies a temperature of $\sim 200 \mathrm{eV}$, as discussed later. Similar data also showed a double peak of proton heating, which correlated with a double peak of x-ray emission from the irradiated hemisphere. This suggests that observation of multiple proton heating peaks in this experiment is due to inhomogeneity of irradiation, which distorts the topology of the Debye sheath and perturbs the local direction of proton acceleration. $^{20}$

\section{Temperature measurements}

The $68 \mathrm{eV}$ emission is optically thick at $0.1 \mu \mathrm{m}$ depth in solid $\mathrm{Al}$, so that the recorded image is from heating close to the rear surface. The rear surface temperature was deduced from the absolute brightness of the XUV Planckian emission. ${ }^{18}$ We compared the time-integrated emission with a one-dimensional (1D) model calculated with the radiationhydrodynamics code LASNEX. ${ }^{21}$ The XUV opacities in these calculations were derived from the super-transition array formalism ${ }^{22,23}$ and also from an in-line, average atom 


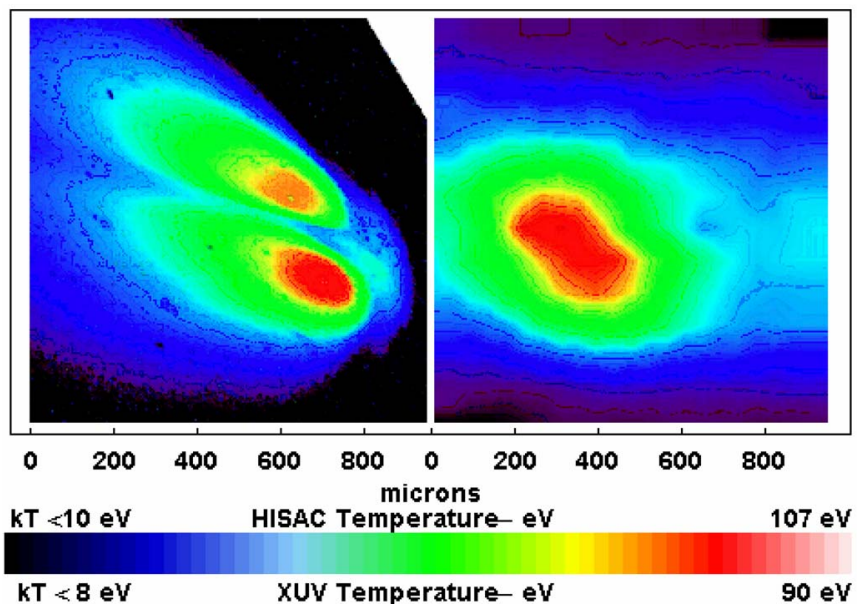

FIG. 3. (Color online) XUV (left) and HISAC (right) comparison of temperature maps of the same shot at $D / r=1.74$ corrected geometrically to have same spatial scale and angle of view normal to the surface.

method. ${ }^{24}$ We found little sensitivity to which opacity model was used. In the 1D model an infinitely wide aluminum slab, of the same thickness as was used in the experiment, was given a range of initial temperatures $T_{0}$ and allowed to expand and cool both adiabatically and radiatively. The calculated, time-integrated, bandpass-integrated intensities, in the same direction with respect to the slab normal as in the experiment, were then compared to the observations. This procedure yields a transformation from XUV intensity to $T_{0}$. The sensitivity of the integrated brightness to the finite target thickness effects is very minor compared to the sensitivity to the initial temperature.

Similar calibration was employed for HISAC as summarized in Ref. 19. Figure 3 compares deduced temperature maps for heating a $100 \mu \mathrm{m}$ thick Al slab with a $D / r=1.74$. Given that HISAC has a limited resolution of $\sim 45 \mu \mathrm{m}$, there is a fair correlation between the two temperature maps. The HISAC image blurs the XUV double spot structure. However the geometrical projection and orientation correction for viewing normal to the target surface cannot remove the apparent plume from the XUV data. However, the orientation, scale, structure, and peak temperature of the two images are otherwise consistent.

The XUV data for 13 shots of the focal plane scan with $100 \mu \mathrm{m}$ thick Al slabs are shown in Fig. 4. The stopping thickness for $3.5 \mathrm{MeV}$ protons normally incident from the front surface is $100 \mu \mathrm{m} \mathrm{Al}$, so the recorded image is dominated by protons of energy close to $3.5 \mathrm{MeV}$. $^{3,11}$ The highest temperature produced at the rear surface of the $100 \mu \mathrm{m}$ foil was $81 \mathrm{eV}$. The temperature data are normalized for shot to shot variance of the laser energy by dividing temperature by the laser energy and multiplying by the mean laser energy. The energy on target was approximately $60 \%$ of the energy from the final amplifier due to losses in the grating compressor and focusing optic. The on-target mean energy was $285 \mathrm{~J}$ with an RMS variation of $47.3 \mathrm{~J}$ (30\% of this energy was within the laser focal spot). Two shots at the $D / r$ ratio of 1.25 did not produce an XUV image. The CCD noise floor puts an upper bound temperature on these shots of $8 \mathrm{eV}$. A Gaussian fit shows the best focusing of the $3.5 \mathrm{MeV}$ protons at a $D / r$ ratio of $1.94 \pm 0.4$. The Poisson error in the temperature is deduced from the number detected quanta in each CCD pixel, Poisson errors for all the shots are less than $1 \mathrm{eV}$, and the error bars are too small to be shown in Fig. 4. The scattered data shown in Fig. 4 are due to systematic errors that we can not control; e.g., the error from the target alignment and variability of the laser focal spot. The temperature profile diameters [full width at half-maximum (FWHM)] were measured in both horizontal and vertical directions and then averaged. This minimizes inclusion of plasma plume expansion in the estimation. For data where there are multiple spots, the FWHM of the heating was deduced from the largest radius points at half the peak intensity. Figure 4 (top) shows the radii as a function of $D / r$. We find a minimum proton plasma jet waist with an average diameter of $206 \mu \mathrm{m}$ at $D / r$ $=1.8 \pm 0.4$. This is significantly wider than was observed with a more uniform and smaller focal spot on the hemi-shell. ${ }^{13}$ In

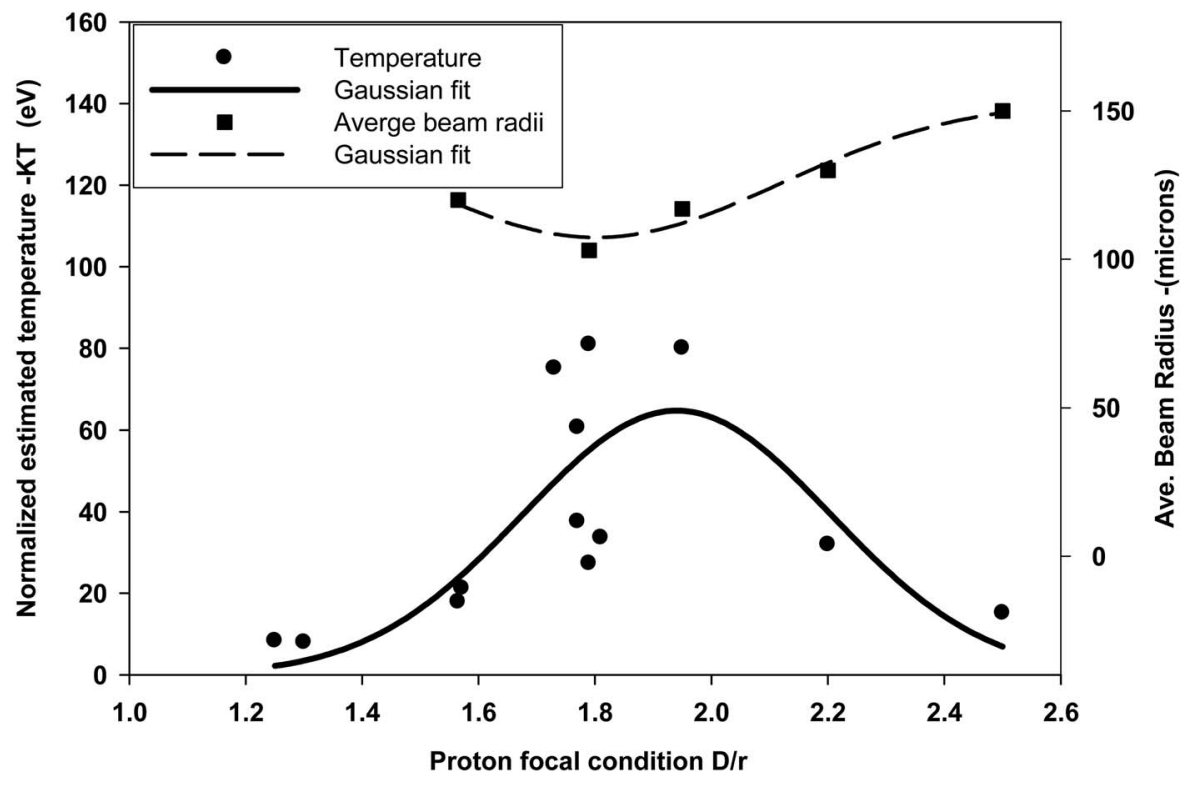

FIG. 4. Filled circles: peak XUV temperatures normalized to the average laser energy with $100 \mu \mathrm{m}$ thick Al targets. Filled squares: averaged radii of the heated spot at half-peak temperature. 


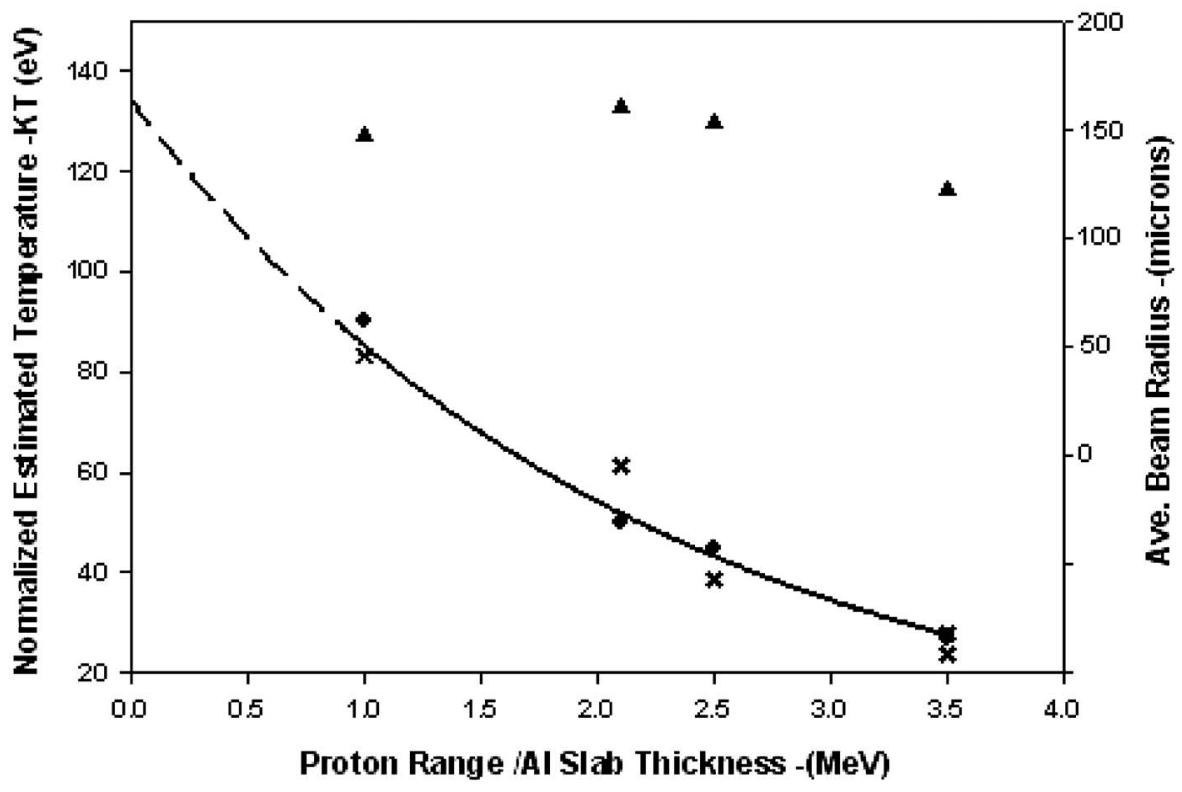

FIG. 5. Crosses: Peak temperatures normalized to the average laser energy at $D / r$ of 1.5 . Line: a simple model fit for a Boltzmann temperature of 2.2 MeV. Dots: a 3D Monte Carlo simulation discussed in the text. Triangles: averaged beam radii.

our work the proton plasma jet was structured and wider and we attribute this to structure in the focal spot pattern, as noted in Sec.III.

A second set of data measured the variation of peak temperature with a change in the Al slab thickness with a fixed $D / r$ ratio of 1.5 . The $D / r$ ratio was chosen prior to the experimental determination of optimal $D / r$ of 1.94 . The Al slab thickness determines the energy band of protons inducing the majority of the heating. ${ }^{3,11}$ We used Al slab thickness (proton energy) of $100 \mu \mathrm{m}(3.5 \mathrm{MeV}), 60 \mu \mathrm{m}$ (2.5 MeV), $47 \mu \mathrm{m}$ $(2.1 \mathrm{MeV})$, and $15 \mu \mathrm{m}(1 \mathrm{MeV})$. The results are shown in Fig. 5.

There is a trend to increased heating at reduced thickness. The highest recorded peak temperature is $83 \mathrm{eV}$ for $15 \mu \mathrm{m}(1 \mathrm{MeV})$ thickness after being normalized to the average laser energy of $359 \mathrm{~J}$.

A simple model fit to the proton data shown as a line in Fig. 5 gives a Boltzmann temperature of $2.2 \mathrm{MeV}$ and thus implies a front surface temperature $\sim 132 \mathrm{eV}$. The model assumes that protons deposit their energy at their Bragg peak that is narrow relative to the slope temperature and that their range increases quadratically with energy.

The very bright spot evident in the x-ray pinhole camera image of Fig. 2 gives another estimation of the front surface temperature. The emission from the front surface of the Al slab is filtered by the Al hemi-shell and the Be filter. The $\mathrm{x}$-ray transmission is appreciable only for $\mathrm{x}$ rays of energy greater than $\sim 900 \mathrm{eV}$, consistent with a front surface temperature of 200 to $300 \mathrm{eV}$ on this shot. The higher temperature may be due to heating by other fast ions and co-moving electrons. Other fast ions with higher charge and mass have a very short range and can cause strong surface heating. Comoving electrons which have much lower kinetic energy and shorter range will contribute their energy to the front surface for thick Al slabs, but will have some effects for very thin Al foils (15 $\mu \mathrm{m} \mathrm{Al} \mathrm{slab} \mathrm{in} \mathrm{our} \mathrm{experiment).}$

Regarding our method of determining temperature via 1D simulation of the XUV emission, some caution must be observed. There are two potentially significant effects, which we have ignored: lateral expansion and initial temperature gradients from the exponential proton spectrum in the solid Al. The protons only heat a finite spot. In the XUV "pictures," this spot is wider than the slab thickness, so the initial expansion is roughly 1D. Nevertheless, the pictures show plume structures apparently expanding away from the initial hot spots. Hence, the XUV camera is looking through the side of the plume and the expansion must be $2 \mathrm{D}$ or $3 \mathrm{D}$, with more adiabatic cooling and a steeper density gradient in the rarefaction.

We performed several calculations to test the model sensitivity to the above effects. The overwhelming sensitivity of the observed integrated intensity is to $T_{0}$ : it scales roughly as $T_{0}^{3}$ in the range of $20-200 \mathrm{eV}$. The lateral expansion effect seen in 2D calculations is almost negligible for the observed spot sizes and $T_{0}$ in the range of $20-60 \mathrm{eV}$; at higher $T_{0}$ the effect increases, reducing the intensity seen for $100 \mathrm{eV}$ by about $50 \%$, still resulting in only a modest temperature error.

\section{MONTE CARLO MODEL OF PROTON HEATING}

To estimate the energy content of the proton plasma jet and its energy spectrum we constructed a 3D Monte Carlo proton transport and dose code with self-consistent heating, explicit equation of state (EOS), and hot stopping powers for the protons. ${ }^{25}$ We used a simple hot stopping model ${ }^{25}$ with a SESAME EOS together with a collisional code kernel. ${ }^{26}$ Following the prescription in Mehlhorn, ${ }^{25}$ a mean ionization potential $\langle I\rangle$ was calculated for an effective $Z$, and the cold stopping powers were adjusted for this effective $Z$ and $\langle I\rangle$ to give the bound stopping in warm material. We assumed a Boltzmann spectrum and a Gaussian transverse intensity. The area $\times$ temperature integral $\int T d a$ in the model was the same as that determined from the XUV images at best focus after these images were transformed to temperature plots. The protons were sent into the target in order of decreasing energy to mimic the time-of-flight characteristic dependence 
laser-driven protons. The protons entered the aluminum at normal incidence. The model temperature and total energy were varied to fit the experimental data in Fig. 5 leading to a $k T$ of $2 \mathrm{MeV}$, in agreement with the simple model, and total proton energy of $5.4 \mathrm{~J}$. The average laser energy on target was $173 \mathrm{~J}$, so the conversion efficiency from laser to proton energy was $3.1 \%$.

\section{SUMMARY}

We have measured the 2D spatial pattern of isochoric heating of $\mathrm{Al}$ foil targets by focused laser-driven proton plasma jets. There is a demonstrated temperature peak and beam waist at 0.94 times the hemisphere radius away from the equatorial plane. Peak temperatures reached $83 \mathrm{eV}$. We deduced the proton energy spectrum to have a Boltzmann temperature of $2 \mathrm{MeV}$ and energy content $3.1 \%$ of the laser energy.

\section{ACKNOWLEDGMENTS}

This project is supported by the US Office of Fusion Energy Sciences under Contract No. AT5015033. This work was performed under the auspices of the U.S. Department of Energy by the University of California, Lawrence Livermore National Laboratory under Contract No. W-7405-Eng-48.

${ }^{1}$ M. Tabak, J. Hammer, M. E. Glinsky, W. L. Kruer, S. C. Wilks, J. Woodworth, E. M. Campbell, M. D. Perry, and R. J. Mason, Phys. Plasmas 1, 1626 (1994).

${ }^{2}$ R. Kodama, P. A. Norreys, K. Mima et al., Nature 412, 798 (2001).

${ }^{3}$ R. A. Snavely, M. H. Key, S. P. Hatchett et al., Phys. Rev. Lett. 85, 2945 (2000).
${ }^{4}$ M. Roth, T. E. Cowan, M. H. Key et al., Phys. Rev. Lett. 86, 436 (2001).

${ }^{5}$ M. H. Key, R. R. Freeman, S. P. Hatchett, A. J. Mackinnon, P. K. Patel, R. A. Snavely, and R. B. Stephens, Fusion Sci. Technol. 49, 440 (2006).

${ }^{6}$ G. Pretzler, A. Saemann, A. Pukhov et al., Phys. Rev. E 58, 1165 (1998).

${ }^{7}$ M. Zepf, M. Castro-Colin, D. Chambers et al., Phys. Plasmas 3, 3242 (1996).

${ }^{8}$ K. Codling and L. J. Frazinski, Contemp. Phys. 35, 243 (1994).

${ }^{9}$ T. Ditmire, J. Zweiback, V. P. Yanovsky, T. E. Cowan, G. Hays, and K. B. Wharton, Nature 398, 489 (1999).

${ }^{10}$ K. Krushelnick, E. L. Clark, M. Zepf et al., Phys. Plasmas 7, 2055 (2000).

${ }^{11}$ S. P. Hatchett, C. G. Brown, T. E. Cowan et al., Phys. Plasmas 7, 2076 (2000).

${ }^{12}$ J. C. Olson, H. A. Davis, and W. J. Waganaar, IEEE Trans. Plasma Sci. 24, 65 (1996).

${ }^{13}$ P. K. Patel, A. J. Mackinnon, M. H. Key, T. E. Cowan, M. E. Foord, M. Allen, D. F. Price, H. Ruhl, P. T. Springer, and R. Stephens, Phys. Rev. Lett. 91, 125004 (2003).

${ }^{14}$ R. Ramis and J. Ramirez, Nucl. Fusion 44, 720 (2004).

${ }^{15}$ M. Temporal, J. J. Honrubia, and S. Atzeni, Phys. Plasmas 9, 3098 (2002).

${ }^{16}$ S. Atzeni, M. Temporal, and J. J. Honrubia, Nucl. Fusion 42, L1 (2002).

${ }^{17}$ Y. Kitagawa, Y. Sentoku, S. Akamatsu et al., Phys. Plasmas 9, 2202 (2002).

${ }^{18}$ P. Gu, B. Zhang, M. H. Key et al., Rev. Sci. Instrum. 77, 113101 (2006).

${ }^{19}$ R. Kodama, K. Okada, and Y. Kato, Rev. Sci. Instrum. 70, 625 (1999).

${ }^{20}$ J. Fuchs, T. E. Cowan, P. Audebert et al., Phys. Rev. Lett. 91, 255002 (2003).

${ }^{21}$ G. B. Zimmerman and W. L. Kruer, Comments Plasma Phys. Controlled Fusion 2, 51 (1975).

${ }^{22}$ A. Bar-Shalom, J. Oreg, W. H. Goldstein, D. Shvarts, and A. Zigler, Phys. Rev. A 40, 3183 (1989).

${ }^{23}$ T. Blenski, A. Grimaldi, and F. Perrot, Phys. Rev. E 55, R4889 (1997).

${ }^{24}$ W. A. Lokke and W. H. Grasberger, in Lawrence Livermore National Laboratory Report No. UCRL-52276, 1977. Copies may be ordered from National Technical Information Service, Springfield, VA 22161

${ }^{25}$ T. A. Mehlhorn, J. Appl. Phys. 52, 6522 (1981).

${ }^{26}$ J. F. Ziegler, J. P. Biersack, and U. Littmark, The Stopping and Range of Ions in Solids (Pergamon, New York, 1985). 